\title{
Protective role of oral antioxidant supplementation in ocular surface of diabetic patients
}

\author{
V Peponis, M Papathanasiou, A Kapranou, C Magkou, A Tyligada, A Melidonis, \\ T Drosos, N M Sitaras
}

Br J Ophthalmol 2002;86:1369-1373

See end of article for authors' affiliations

Correspondence to: Nikolaos M Sitaras, MD $\mathrm{PhD}$, Department of Pharmacology, Medical School, University of Athens, 75 Mikras Asias Street, Athens 11527 Greece; nisitpav@hol.gr

Accepted for publication 2 July 2002

\begin{abstract}
Aim: To investigate the effect of vitamin $C$ and $E$ supplementation in the levels of nitrite, nitric oxide (NO) related metabolite, and ocular surface parameters in diabetic patients.

Methods: 50 patients with non-insulin dependent diabetes mellitus were given vitamin C (1000 $\mathrm{mg} /$ day) and vitamin E (400 IU/day) supplementation for 10 days. Nitrite levels in tears were measured by photometric determination before and after vitamin supplementation. Tear function parameters (Schirmer test I, BUT, ocular ferning test) and brush cytology analysis of the conjunctival epithelium were also evaluated.

Results: Nitrite levels were found to be significantly reduced ( $p<0.05)$ after 10 days of vitamin $C$ and E supplementation. Improved values for Schirmer test, BUT test, and ocular ferning test were also found. Goblet cell density and grading of squamous metaplasia showed a significant improvement.

Conclusions: Oxidative stress and free radical production are elevated in diabetes mellitus. Antioxidants, such as vitamin $\mathrm{C}$ and vitamin $\mathrm{E}$, probably have an important role in reducing the oxidative damage produced by nitric oxide and other free radicals and improving the ocular surface milieu.
\end{abstract}

D iabetes mellitus is associated with a number of ocular complications which can lead even to blindness. The study of ocular surface manifestations during the course of diabetes mellitus has increased in recent years. For example, $47 \%-67 \%$ of diabetic patients have primary corneal lesions during their lifetime. ${ }^{1}$ In addition many diabetic patients complain of dry eye symptoms, indicating a clear role for tear film abnormalities. ${ }^{2}$

The ocular surface is relatively unprotected and constantly exposed to radiation, atmospheric oxygen, environmental chemicals and physical insults, resulting in the generation of reactive oxygen species (ROS) which are thought to contribute to ocular damage. ${ }^{3}$ Vitamin $\mathrm{C}$ (ascorbic acid) is found in high concentration in the eye and is thought to be a primary substrate in ocular protection. ${ }^{4}$ Moreover, most studies have found that people with diabetes mellitus have circulating ascorbic acid concentrations at least 30\% lower than people without diabetes mellitus.

Nitric oxide (NO) serves a wide variety of functions in various biological systems, both in intracellular compartments as a second messenger that responds to activation of plasma membrane receptors and in extracellular compartments as a paracrine factor that carries information between cells. ${ }^{\circ}$

Nitric oxide is synthesised from L-arginine by three distinct isoforms of nitric oxide synthase (NOS) which are products of three different genes. ${ }^{7}$ The three isoforms of NOS are classified as follows: neuronal NOS (NOS1), inducible NOS (NOS2), and endothelial NOS (NOS3). ${ }^{8}$ Nitric oxide is constantly produced by NOS1 and NOS3, which are activated via the calcium/ calmodulin complex. By contrast, the activity of NOS2 is independent of calcium and capable of generating large amounts of NO in the presence of immunological and inflammatory stimuli. ${ }^{8}$

Nitric oxide has been implicated in various physiological and pathological processes in the eye. It contributes to the regulation of aqueous humour dynamics, retinal neurotransmission, phototransduction, regulation of retinal vascular tone, ocular inflammatory diseases (uveitis, retinitis), degenerative diseases (glaucoma, retinal degeneration), allergic eye disease, and diabetic retinopathy. ${ }^{6-8}$
Induction of iNOS (inducible NOS) by cytokines leads to the production of large amounts of NO which mediate the destructive responses in ocular inflammation. ${ }^{6}$ Peroxynitrite $\left(\mathrm{ONOO}^{-}\right)$formed by the reaction between nitric oxide and superoxide $\left(\mathrm{O}_{2}^{-}\right)$is a powerful oxidant capable of causing tissue injury.

There are observations that high glucose levels lead to increased NOS expression and increased NOS activity. ${ }^{10}$ This could probably be attributed to increased oxidative stress that is evident in diabetes mellitus. ${ }^{9}$

The above observations raise the question of whether antioxidant supplementation could modify NO levels as well as tear film characteristics in diabetes mellitus.

In this study the potential effect of oral vitamins $\mathrm{C}$ and $\mathrm{E}$ on NO levels and various clinical and cytological parameters of tear film and ocular surfaces in diabetic patients was investigated.

\section{PATIENTS AND METHODS}

Ninety seven eyes of 50 patients diagnosed with non-insulin dependent diabetes mellitus (NIDDM) were enrolled in this study from the department of ophthalmology and the diabetes centre of the general hospital of Piraeus. Patients characteristics are summarised in Table 1.

\begin{tabular}{ll}
\hline Table 1 & Patient characteristics \\
\hline Age (years) & $63.64(10.33)^{*}$ \\
Sex (M/F) & $17 / 33$ \\
Duration of diabetes (year) & $10.85(9.08)^{*}$ \\
Therapy (hypogl/insul) & $35 / 15$ \\
Smoke (yes/no) & $7 / 43$ \\
$\mathrm{HBA}_{1 \mathrm{C}}(\%)$ & $8.56(1.58)^{*}$ \\
Neuropathy (no/yes) & $26 / 24$ \\
Retinopathy (O/1/2/3) $\dagger$ & $14 / 18 / 12 / 6$ \\
\hline *Mean (SD) & \\
†O=no diabetic retinopathy, $1=$ background retinopathy 2 $=$ \\
non-proliferative retinopathy, $3=$ proliferative retinopathy. \\
\hline
\end{tabular}


Exclusion criteria from the study were (a) any systemic disease other than NIDDM, (b) medications other than hypoglycaemic drugs or insulin that could interfere with tears and ocular surface parameters, (c) topical eye medications within the past 6 months, (d) history of ophthalmic surgical or laser procedures, (e) eye diseases other than dry eye and/or diabetic retinopathy, (f) history of taking vitamin supplements. The diagnosis of peripheral neuropathy was based on abnormal nerve conduction velocity test results and the presence of symptoms and signs of diabetic polyneuropathy such as neuropathic ulcers, dysaesthesias, paraesthesias, and abnormal deep tendon reflexes. The status of diabetic retinopathy was assessed by fundus examination and, if necessary, by fluorescein angiography.

Patients entered in the study had a careful slit lamp examination and a detailed ocular and systemic medical history. The dietary intakes of vitamin $\mathrm{C}$ and $\mathrm{E}$ were assessed carefully.

Immediately after accrual patients were given a combination of vitamin C (1000 mg/day) and E (400 IU/day) (Nutrition Headquarters, IL, USA) for a period of 10 days. Vitamin C can enhance the activity of vitamin E by reducing the tocopheroxyl radical and thereby restoring the radical scavenging activity of vitamin E. ${ }^{11}$ All the measurements were done on days 1 and 11 . Any adverse reaction was immediately reported.

The study was carried out in accordance with the principles of the Declaration of Helsinki. The subjects entered the study after informed consent was given, following a full explanation describing the procedures and the nature of the study.

\section{Methods}

All measurements were done by the same investigator in a quiet, dimly lit examination room of relatively constant temperature and humidity.

\section{Schirmer test}

A conventional Schirmer I test without anaesthesia was performed by placing the short portion of a folded Schirmer test strip (Ciba-Vision, Switzerland) over the lower lid margin and measuring the amount of wetting in mm after 5 minutes. Values of less than $10 \mathrm{~mm}$ of wetting are considered abnormal.

\section{Fluorescein tear break up time (BUT)}

The standard tear film BUT measurement was performed using moistened fluorescein strips (Chauvin Pharmaceuticals Ltd, Essex, UK). The time interval between the last complete blink and the appearance of the first black spot in the preocular tear film was measured three times and the mean value of the measurements was calculated. A BUT value of less than 10 seconds was considered abnormal.

\section{Ocular ferning test}

A glass capillary tube was used to collect the tear fluid from the lateral part of the inferior tear meniscus. The sample was dropped onto a light microscopy slide, allowed to dry at room temperature for 5-10 minutes, and then observed by phase contrast light microscopy at a magnification of $\times 40-100$. On the basis of its average appearance each sample was assigned to one of the four types in the Rolando classification. ${ }^{12}$

Ronaldo classified tear film crystallisation into four types (grade I-IV): grade I consists of large, homogeneous ferns and uniform closely branching arborisation; in grade II ferns are smaller and sparsely distributed; in grade III arborisation is scarcely present with many empty spaces, and in grade IV ferning is totally absent and clusters of mucus may be present.

Types III and IV are considered abnormal. All the ferning specimens were evaluated by two investigators in a masked fashion.

\section{Conjunctival brush cytology}

A disposable brush (Accelon R Multi Biosampler, Medscand, Malmö, Sweden) was used. After topical installation of anaes- thetic the temporal bulbar conjunctiva was scraped by gentle rotations of the brush under slit lamp observation. Care was taken not to touch any part of the surface except that being examined. The collected material was smeared on slides by rotations of the brush and then fixed with Cytospray. The brush samples were stained with periodic acid Schiff (PAS) and Papanicolaou stain and were evaluated for the number of goblet cells and the presence of squamous metaplasia of conjunctival epithelial cells with a light microscope (Zeiss, Germany) at a magnification of $\times 10-40$ according to a grading system originally described by Nelson ${ }^{13}$ and slightly modified by Rojas et al..$^{14}$ All cytology specimens were evaluated by two investigators in a masked fashion.

\section{Nitric oxide measurements}

A volume of $100 \mu \mathrm{l}$ water for injection was installed in the lower conjunctival fornix and after three forced blinkings lavage fluid was collected using a micropipette and avoiding any contact with the ocular surface. Nitrite concentration, the stable end product of NO, was measured using the Griess reaction. ${ }^{15}$ Briefly, the samples were mixed with the same volume of Griess reagent (mixture of one part of $0.1 \%$ naphthylethylenediamine dihydrochloride in water and one part of $1 \%$ sulfanilamide in $5 \%$ hydrochloric acid) and allowed to react at room temperature for 30 minutes. The concentrations of nitrite were then determined photochemically by measuring absorbance at $540 \mathrm{~nm}$.

The order of performing the tests was as follows. Lavage fluid for nitrite determination was taken first in order to avoid any ocular irritation that could interfere with subsequent measurements; ocular ferning test; BUT test; Schirmer test; brush cytology. An adequate time interval (15-20 minutes) was left between the tests to prevent one procedure interfering with the results of subsequent tests.

\section{Statistical analysis}

In order to compare the results before and after treatment parametric Student's $t$ tests were used for data which followed normal distribution and distribution free statistical tests (Wilcoxon sign rank test) for non-normal data. Regression analysis was used to test the effects of various factors (baseline characteristics such as age, duration of diabetes, smoking habits, status of peripheral neuropathy, severity of retinopathy) on the continuous variables. Spearman's correlation analysis was used to explore correlations between the vitamin induced changes in the parameters that were evaluated. A probability level less than $5 \%$ was considered statistically significant.

\section{RESULTS}

\section{Schirmer test}

The mean Schirmer test value in the diabetic patients before vitamin supplementation was 12.94 (SD 6.81) $\mathrm{mm}$ and after treatment was $15.86(6.44) \mathrm{mm}$. The difference was statistically significant $(\mathrm{p}<0.001)$. Seventy eight per cent $(95 \%$ confidence interval (95\% CI): 64 to 88 ) of patients who received treatment showed an improvement in Schirmer test values; $77 \%$ of patients with values less than $10 \mathrm{~mm}$ before treatment and $78 \%$ of patients with values more than $10 \mathrm{~mm}$ before treatment showed improvement suggesting an equal effect in both groups.

A mild but statistically significant correlation was found between the differences in Schirmer test values before and after supplementation and the differences in BUT values (Spearman's correlation coefficient, $r_{\mathrm{s}}=0.384 ; \mathrm{p}=0.006$ ). Another significant correlation was observed between the differences in Schirmer test values and the differences in nitrite levels $\left(r_{\mathrm{s}}=0.388 ; \mathrm{p}=0.008\right)$. 


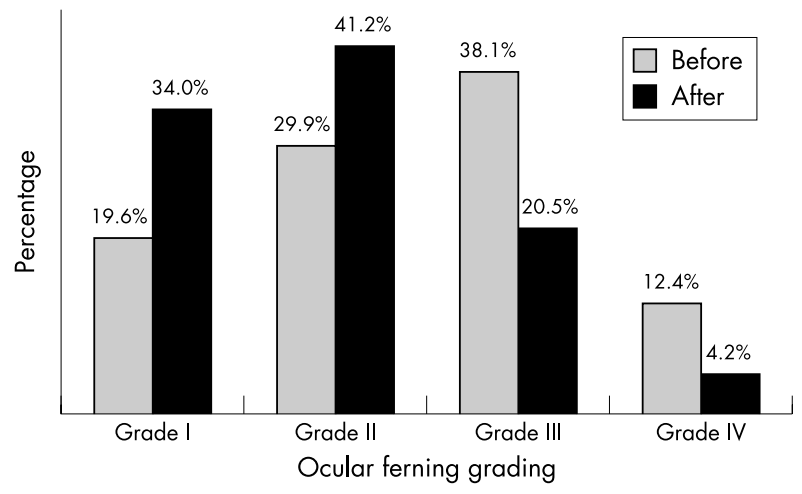

Figure 1 Ocular ferning grading (evaluated according to Rolando ${ }^{12}$ ) in diabetic patients before and after vitamin $C$ and $E$ supplementation.

\section{BUT test}

The median BUT test value in the diabetic patients before vitamin supplementation was 10.25 seconds (range 5.5-25 seconds) and after treatment was 12.75 seconds (range 8-27 seconds). The difference was statistically significant $(\mathrm{p}=0.001)$. All the patients $(\mathrm{n}=19)$ with BUT less than $10 \mathrm{sec}-$ onds showed improvement compared to $60 \%$ of patients with BUT of more than 10 seconds.

A mild but statistically significant correlation was found between the differences in BUT test values before and after supplementation and the differences in ferning test values $\left(r_{\mathrm{s}}=0.438 ; \mathrm{p}=0.002\right)$. Another significant correlation was found between the differences in BUT test values and the differences in goblet cells densities $\left(r_{\mathrm{s}}=0.459 ; \mathrm{p}=0.001\right)$.

\section{Ocular ferning test}

The average grade of the ferning test was 2.43 (SD 0.85) before supplementation and 1.96 (0.77) after supplementation ( $p$ $<0.001)$, which shows an improvement. A total of $64 \%(95 \%$ CI: 49 to 77) of patients showed improvement (defined as grade before $>$ grade after supplementation) (see also Fig 1).

Patients with duration of diabetes $>15$ years showed less improvement (mean value $0.06(0.77)$ ) compared to patients with duration of diabetes $<15$ years (mean value $0.64(0.63)$ ); $\mathrm{p}$ value $=0.018$. Additionally, patients $>65$ years old showed less improvement (mean value $0.19(0.76)$ ) compared to patients $<65$ years old (mean value $0.7(0.6)$ ); $p$ value $=0.008$.

A mild but statistically significant correlation was found between the differences in ferning test values before and after supplementation and the differences in goblet cell densities. $\left(r_{\mathrm{s}}=0.458 ; \mathrm{p}=0.001\right)$.

\section{Nitrite concentration measurements}

The mean nitrite value in the diabetic patients before vitamin supplementation was 3.24 (SD 1.23) $\mu \mathrm{M}$ and $2.21(0.85) \mu \mathrm{M}$ after treatment. The difference was statistically significant ( $p<0.001$ ); $78 \%$ (95\% CI: 63 to 89) of treated patients showed a reduction in nitrite values after supplementation.

Using univariate regression analysis we showed that the reduction in nitrite was greater in patients with proliferative diabetic retinopathy compared to patients without diabetic retinopathy $(\mathrm{p}=0.001)$.

\section{Brush cytology analysis}

\section{Goblet cell density}

The average goblet cell densities were 49 cells/per unit field (range 19-259) before vitamin supplementation and 58 cells/ per unit field (range 18-178) after supplementation with the difference being statistically significant $(p=0.003) ; 76 \%$ of patients showed an increase in goblet cell densities (95\% CI: 61 to 86 ). We also found that patients exhibiting signs of diabetic neuropathy showed less increase in goblet cell densities (mean value 1.2 (18.7) cells/per unit field) compared to patients without diabetic neuropathy (mean value 15.11 (34.43) cells/per unit field); $p=0.012$.

Using multivariate regression analysis we showed that the increase in goblet cell densities was adversely affected by age $(p=0.027)$ and duration of diabetes $(p=0.035)$.

\section{Squamous metaplasia}

The average grade of squamous metaplasia was 1.1 (0.42) before supplementation and 0.9 (0.49) after supplementation $(\mathrm{p}=0.01)$ which also shows a significant improvement; $44 \%$ (95\% CI: 30 to 58) of patients showed improvement (defined as grade before $>$ grade after supplementation).

We also found that diabetic neuropathy adversely affects the improvement in squamous metaplasia stage (Pearson's $\chi^{2}$, $\mathrm{p}=0.042$ ).

\section{DISCUSSION}

Although the Diabetes Control and Complications Trial has identified hyperglycaemia as a significant risk factor for the development of diabetic complications, ${ }^{16}$ the full spectrum of the pathophysiological mechanisms of chronic diabetic complications hasn't been thoroughly elucidated. Some equally tenable hypotheses for the origin of complications are oxidative stress damage, ${ }^{17} 18$ advanced glycation end products (AGE) hypothesis, ${ }^{19} 20$ aldose reductase pathway, ${ }^{21}$ reductive stress (pseudohypoxia), ${ }^{22}$ true hypoxia, ${ }^{23}$ carbonyl stress, ${ }^{24}$ altered lipoprotein metabolism, ${ }^{25}$ increased protein kinase C activity, ${ }^{26}$ and altered growth factors, ${ }^{27}$ and cytokine ${ }^{28}$ activities.

The various hypotheses overlap and intersect with one another: AGE formation and altered polyol pathway activity may lead to oxidative stress, oxidative stress may accelerate AGE formation, reductive stress may lead to activation of protein kinase $\mathrm{C}$ activity, AGEs may induce growth factors and cytokines production, and so on. ${ }^{29}$

Oxidative stress has been defined as a disturbance in the balance between the production of reactive oxygen species (especially free radicals) and the antioxidant defence status which may lead to tissue injury. ${ }^{30}$ Oxidative stress is an acknowledged pathogenetic mechanism in diabetic complications. Beyond that it appears that a number of antioxidant defence systems are compromised in diabetic individuals. ${ }^{31}$ These include decrease in plasma ascorbic acid levels, ${ }^{32} 33$ intracellular deficiency of ascorbic acid (cellular scurvy), ${ }^{34}$ and decrease in cellular vitamin E levels. ${ }^{35}$ Moreover, ocular tissues probably have a higher free radical activity than any other organ, mainly because of ultraviolet exposure. ${ }^{36}$

Higher levels of NO were found in the aqueous humour of diabetic patients and this may induce inflammatory reactions that cause cell damage. ${ }^{37}$ Biswas et al have reported that in the resting state the levels of $\mathrm{NO}$ were higher in diabetic compared to normal polymorphonuclear leucocytes. ${ }^{38}$ Macrophages have been shown to increase NO production in diabetes. ${ }^{39}$ The large amounts of NO which are produced may occur during iNOS mediated NO release. ${ }^{40}$ ROS mediate signal transduction, including activation of the transcription factor NF-kB (nuclear factor $\mathrm{kB}$ ), which is crucial for the inducible expression of genes involved in proinflammatory cytokines production (IL-1, IL-6, TNF- $\alpha$ ). ${ }^{41}$ The cytokines could then activate DNA directed mRNA synthesis that induces synthesis of iNOS. ${ }^{42}$ Additionally, the reaction of nitric oxide with superoxide $\left(\mathrm{O}_{2}^{-}\right)$leads to the formation of peroxynitrite, a potent oxidant which contributes to ocular inflammation. ${ }^{43}$

Our results show that vitamin $\mathrm{C}$ and $\mathrm{E}$ supplementation decreases NO levels in the lavage fluid from the ocular surface of diabetic patients towards the levels that we have found in normal healthy subjects $(2.11(0.93) \mu \mathrm{M}$, range $1.13-2.97 \mu \mathrm{M}$; unpublished data). We suggest that the antioxidant activity of these compounds results in a decrease in the oxidative burden 
in the ocular surface that could lead to elimination of NO and its cytotoxic effects. We suggest that this could be achieved through downregulation of the cytokine induced activation of iNOS. Interestingly, patients with proliferative diabetic retinopathy showed the greater reduction in nitrite levels. These patients had the greatest pretreatment values of nitrite and benefit more from vitamin supplementation. Recently Yilmaz et al found elevated NO levels in the vitreous of diabetic patients with proliferative diabetic retinopathy compared to controls. ${ }^{44}$ We haven't found significant differences in NO levels between smokers and non-smokers although smoking is a well known oxidative factor.

Our results also demonstrate that the orally administered antioxidant supplements improve the tear film stability, tear secretion, and health of the ocular surface. These results are in accordance with previous reports. ${ }^{45} 46$

There are several mechanisms that could explain our findings. The antioxidant properties of vitamins $C$ and $E$ could protect the ocular surface from free radical attack and preserve the integrity of the ocular epithelium. The above mentioned reduction of $\mathrm{NO}$ and probably peroxynitrite could abolish the cytotoxic effects of these compounds. Additionally, vitamins A, C, and E are needed for cell differentiation, development, and maintenance. ${ }^{47}$ Deficiencies of these vitamins in animals caused loss of goblet cells in the conjunctiva and abnormal chromatin distribution in the nucleus of epithelial cells. ${ }^{47}$ Tseng et al speculated that inflammation and loss of vascularisation are two possible mechanisms for loss of goblet cells in various ocular surface disorders. ${ }^{48}$ On the other hand vitamin $\mathrm{C}$ could have an endogenous anti-inflammatory role in the eye. ${ }^{49}$ Human tears are rich in vitamin $\mathrm{C}$ which acts protectively for the ocular tissues. ${ }^{50}{ }^{51}$ Certainly the nutritional influences on tear film composition and physiology are complex.

We also found that patients with diabetic neuropathy are less likely to benefit from vitamin supplementation regarding the improvement in goblet cell counts and squamous metaplasia grade. These patients had probably decreased corneal sensitivity as a manifestation of their diabetic neuropathy. ${ }^{52}$ The subsequent decrease of neurotrophic effects of the trigeminal sensory nerve on the cornea and the conjunctiva may be responsible for these ocular surface changes. ${ }^{53}$ As a consequence these patients had lower initial values for goblet cells and squamous metaplasia and less improvement compared to the patients without neuropathy. Age and duration of diabetes, which are also correlated with decreased corneal sensitivity, adversely affected the changes in goblet cell counts.

The improved BUT values, which suggest increased tear film stability, are clearly correlated with the number of goblet cells. However, because tear film stability is essential for the health of the ocular surface we couldn't determine if BUT values improved as a result of increased mucin production from goblet cells ${ }^{48}$ or vice versa.

Schirmer test values were found to be significantly increased after the treatment period. Although this test is considered a rough screening test for the detection of tear production when performed as a standardised procedure with the same investigator, as in our study it could provide valuable information. Shreeve et al ${ }^{54}$ proposed that lacrimal gland secretion is promoted by micronutrients (zinc, magnesium) and vitamins (C, B6, and niacin). The apparent improvement in Schirmer test values and the correlation with BUT improvement could also reflect the water retentive properties of mucins, ${ }^{55}$ resulting in an increased precorneal residence time, $^{55}$ increased corneal wettability, and reduced tear evaporation from the ocular surface. ${ }^{56}$

Tear ferning patterns depend on the interaction between electrolytes, protein, and mucin macromolecules. ${ }^{57}$ We found a mild correlation between the changes in goblet cells densities and ferning test values. The increased number of goblet cells with subsequent production of mucin probably accounts for the improvement in ocular ferning grade after vitamin supplementation. This could also account for the minor changes in ferning observed in patients $>65$ years old and with a duration of diabetes $>15$ years.

We concluded in this study that oral vitamin $\mathrm{C}$ and $\mathrm{E}$ supplementation could have a protective role in the ocular surface of diabetic patients, leading to improvement in various clinical and cytological parameters as well as a significant reduction in potentially hazardous nitric oxide levels. Although the effect of vitamins was beneficial, the short duration of supplementation does not allow us to draw conclusions for the effects of prolonged vitamin administration. This is a subject of further investigation.

\section{Authors' affiliations}

V Peponis, M Papathanasiou, Department of Ophthalmology, General Hospital of Piraeus "Tzaneion", Greece

V Peponis, C Magkou, A Tyligada, N M Sitaras, Department of Pharmacology, Medical School, University of Athens, Greece A Kapranou, Department of Pathology, General Hospital of Piraeus

"Tzaneion", Greece

A Melidonis, Diabetes Center, General Hospital of Piraeus "Tzaneion", Greece

T Drosos, "Pammakaristos" General Hospital, Athens, Greece

\section{REFERENCES}

1 Schultz RO, Van Horn DL, Peters MA, et al. Diabetic keratopathy. Trans Am Ophthalmol Soc 1981;79:180-99.

2 Goebbles $M$. Tear secretion and tear film function in insulin dependent diabetics. Br J Ophthalmol 2000;84:19-21.

3 Knight JA. Reactive oxygen species and the neurodegenerative disorders. Ann Clin Lab Sci 1997:27:1 1-25.

4 Rose RC, Richer SP, Bode AM. Ocular oxidants and antioxidant protection. Proc Soc Exp Biol Med 1998;217:397-407.

5 Will JC, Byers T. Does diabetes mellitus increase the requirement for vitamin C? Nutrition Rev 1996:54:193-202.

6 Becquet F, Courtois Y, Goureau O. Nitric oxide in the eye: multifaceted roles and diverse outcomes. Surv Ophthalmol 1997;42:71-82.

7 Goldstein I, Ostwald P, Roth S. Minireview. Nitric oxide: a review of its role in retinal function and disease. Vis Res 1996;36:2979-94.

8 Schmetterer L, Polak K. Role of nitric oxide in the control of ocular blood flow. Prog Retin Eye Res $2001 ; 20: 823-47$.

9 Rösen P, Nawroth PP, King G, et al. The role of oxidative stress in the onset and progression of diabetes and its complications: a summary of a Congress Series sponsored by UNESCO-MCBN, the American Diabetes Association and the German Diabetes Society. Diabetes Metabol Res Rev 2001;17:189-212.

10 Cosentino F, Hishikawa K, Katusic ZS, et al. High glucose increases nitric oxide synthase expression and superoxide anion generation in human aortic endothelial cells. Circulation 1997;96:25-8.

11 Packer L. Vitamin C and redox cycling antioxidants. In: Packer L, Fuchs , eds. Vitamin $C$ in health and disease. New York: Marcel Dekker, 1997:95-121.

12 Rolando $M$. Tear mucus ferning test in normal and keratoconjunctivitis sicca eyes. Chibert Int J Ophthalmol 1984;2:32-41.

13 Nelson DJ. Cellulose acetate impressions of the ocular surface. Dry eye states. Arch Ophthalmol 1983;101:1869-72.

14 Rojas MV, Rodriguez MT, Blanco JA, et al. Impression cytology in patients with keratoconjuctivitis sicca. Cytopathology 1993;4:347-55.

15 Green LC, Warner DA, Glogowski J, et al. Analysis of nitrate, nitrite and $(15 \mathrm{~N})$ nitrate in biological fluids. Anal Biochem 1982;126:131-8.

16 The DCCT Research Group. The effect of intensive diabetes treatment on the development and progression of long-term complications in insulin-dependent diabetes mellitus: the Diabetes Control and Complications Trial. N Engl J Med 1993;329:977-86.

17 Giugliano D, Cerriello A, Paolisso G. Oxidative stress and diabetic vascular complications. Diabetes Care 1996:19:257-67.

18 Baynes JW, Thorpe SR. The role of oxidative stress in diabetic complications. Curr Opin Endocrinol 1997;3:277-84.

19 Brownlee M. Advanced protein glycosylation in diabetes and aging. Ann Rev Med. 1996;46:223-34.

20 Vlassara H. Recent progress in advanced glycation end products and diabetic complications. Diabetes 1997;46(Suppl 2):S19-25.

21 Hotta N. New approaches for treatment in diabetes: aldose reductase inhibitors. Biomed Pharmacother 1995;49:232-42.

22 Ido Y, Kilo C, Williamson JR. Cytosolic NADH/NAD+, free radical and vascular dysfunction in early diabetes mellitus. Diabetologia 1997:40(Suppl 2):S1 15-17.

23 Cameron NE, Cotter MA. Metabolic and vascular factors in the pathogenesis of diabetic neuropathy. Diabetes 1997;46 (Suppl 2): $531-7$.

24 Lyons TJ, Jenkins AJ. Glycation, oxidation and lipoxidation in the development of the complications of diabetes: a carbonyl stress hypothesis. Diabetes Rev 1997;5:365-91. 
25 Witztum JL. Role of modified lipoproteins in diabetic macroangiopathy Diabetes 1997;46 (Suppl 2):S1 12-14.

26 Ishii H, Daissuke K, King GL. Protein kinase $C$ activation and its role in the development of vascular complications in diabetes mellitus. J Mol Med 1998:76:21-31.

27 Pfeiffer A, Schatz H. Diabetic microvascular complications and growth factors. Exp Clin Endocrinol Diabetes 1994;103:7-14.

28 Sharma K, Ziyadeh FN. Biochemical events and cytokine interactions linking glucose metabolism to the development of diabetic nephropathy. Sem Nephrol 1997; 17:80-92

29 Baynes JW, Thorpe SR. Role of oxidative stress in diabetic complications. A new perspective on an old paradigm. Diabetes 1999;48: 1-9.

30 Halliwell B. Free radicals, antioxidants and human disease: curiosity, cause or consequence. Lancet 1994;344:721-4.

31 Hunt JV. Ascorbic acid and diabetes mellitus. In: Harris RJ, ed. Subcellular biochemistry. Vol 25. Ascorbic acid: biochemistry and biomedical cell biology. New York: Plennum Press, 1996:369-405

32 Jennings $\mathbf{P E}$, Chirico $\mathrm{S}$, Jones $\mathrm{AF}$, et al. Vitamin $\mathrm{C}$ metabolites and microangiopathy in diabetes mellitus. Diabetes Res 1987:6:151-4.

33 Som S, Basu D, Mukheriee S, et al. Ascorbic acid metabolism in diabetes mellitus. Metabolism 1981;30:572-7.

34 Welch RW, Bergsten P, Butler JD, et al. Ascorbic acid accumulation and transport in human fibroblasts. Biochem J 1993;294:505-10.

35 Karpen C, Cataland S, Orisio TM, et al. Interaction of platelet vitamin E and thromboxane synthesis in type 1 diabetes mellitus. Diabetes 1984;33:239-43.

36 Florence TM. The role of free radicals in disease. Aust NZ J Ophthalmol 1995:23:3-7.

37 Chiou SH, Chang CJ, Chou CK, et al. Increased nitric oxide levels in aqueous humor of diabetic patients with neovascular glaucoma. Diabetes Care 1999;22:861-2.

38 Biswas SK, Bhelwa AP, Upadhyay AU, et al. Status of nitric oxide free radicals in diabetic neutrophils: effect of diabetic serum factor on the generation of these species in normal neutrophils and their relation to lysosomal degranulation. Indian J Biochem Biophys 1993;30:293-6.

39 Wu G. Nitric oxide synthesis and the effect of aminoguanidine and NG-monomethyl- L-arginine on the onset of diabetes in the spontaneously diabetic BB rat. Diabetes 1995;44:360-4.

40 Honing MLH, Morrison PJ, Banga JD, et al. Nitric oxide availability in diabetes mellitus. Diabetes Metab Rev 1998;14:241-9.
41 Baeuerle PA, Henkel T. Function and activation of NF-kB in the immune system. Annu Rev Immunol 1994;12:141-79.

42 McCann SM, Licinio M, Wong L, et al. The nitric oxide hypothesis of aging. Exp Gerontol 1998;33:813-26.

43 Allen JB, Keng T, Privalle $\mathrm{CH}$. Nitric oxide and peroxynitrite production in ocular inflammation. Environ Health Perspect 1998;106 (Suppl 5): $1145-9$

44 Yilmaz G, Esser P, Kociek N, et al. Elevated vitreous nitric oxide levels in patients with proliferative diabetic retinopathy. Am J Ophthalmol 2000; 130:87-90.

45 Patel S, Farrell J, Blades KJ, et al. The influence of vitamins and trace element supplements on the stability of the precorneal tear film. Acta Ophthalmol 1993;71:825-9

46 Blades KJ, Patel S, Aidoo KE. Oral antioxidant therapy for marginal dry eye. Eur J Clin Nutr 2001;55:589-97.

47 Amemiya TS. The eye and nutrition. Jpn J Ophthalmol 2000;44:320.

48 Tseng SCG, Hirst LW, Maumenee AE, et al. Possible mechanisms for the loss of goblet cells in mucin-deficient disorders. Ophthalmology 1984;91:545-52.

49 Paterson AP, O'Rourke MC. Vitamin C levels in human tears. Arch Ophthalmol 1987; 105:376-7.

50 Choy CKM, Benzie IFF, Cho P. Ascorbic acid concentration and total antioxidant activity of human tear fluid measured using the FRASC assay. Invest Ophthalmol Vis Sci 2000;41:3293-8.

51 Gogia R, Richer SP, Rose RC. Tear fluid content of electrochemically active components including water soluble antioxidants. Curr Eye Res 1998; 17:257-63

52 Dogru M, Katakami C, Inoue M. Tear function and ocular surface changes in noninsulin-dependent diabetes mellitus. Ophthalmology 2001;108:586-92.

53 Marfurt CF, Echtenkamp SF. The effect of diabetes on neuropeptide content in the rat cornea and iris. Invest Ophthalmol Vis Sci 1995; $36: 1100-6$

54 Shreeve C. Treating the dry eye. Ophthalmic Optician 1982;25:650-1.

55 Nakamura $M$, Hikida $M$, Nakano T, et al. Characterization of water retentive properties of hyaluronan. Cornea 1993;12:433-6.

56 Tsubota K, Yamada M. Tear evaporation from the ocular surface. Invest Ophthalmol Vis Sci 1992;33:2942-50.

57 Golding TR, Baker AT, Rechberger J, et al. X-ray and scanning electron microscopic analysis of the structural composition of tear ferns. Cornea $1994 ; 13: 58-66$ 